\title{
Viral asthma: implications for clinical practice
}

\author{
This article was published in the following Dove Press journal: \\ Journal of Asthma and Allergy \\ 5 July 2010 \\ Number of times this article has been viewed
}

\section{Roger Menendez' Michael D Goldman² \\ 'Allergy and Asthma Center of El Paso, El Paso, TX, USA; ${ }^{2}$ Pulmonary Division, UCLA Gaffen School of Medicine, Los Angeles, CA, USA}

Correspondence: Roger Menendez Allergy and Asthma Research Center of El Paso, 10470 Vista del Sol, El Paso, TX 79925, USA

Tel + I 915 59| 2080

Fax + I915 5912341

Email rmaacep@yahoo.com

\begin{abstract}
The natural history of asthma appears to be driven primarily by the timing and duration of viral respiratory infections. From the very high rate of infections in childhood, to the more sporadic pattern seen in adults, the cycle of acute injury followed by an inefficient repair process helps explain the clinical patterns of asthma severity currently recognized by asthma guidelines. Why the asthmatic host responds to viral injury in a particular way is largely a mystery and the subject of intense investigation. The role of viruses in asthma extends not just to intermittent but to persistent disease, and to both the atopic as well as nonatopic phenotypes. Future therapeutic strategies should include primary prevention via the development of antiviral innate immunity-enhancing vaccines, as well as secondary prevention via the use of antiviral agents, or immunomodulators designed to boost the antiviral response or interrupt the proinflammatory cascade.
\end{abstract}

Keywords: asthma, rhinoviruses, exacerbations, epidemiology, phenotypes, clinical trials

\section{Introduction}

In the last few years, there has been a shift in interest within the asthma community from focusing on what features all asthmatics have in common, to the differences that set them apart. ${ }^{1}$ We now speak routinely of asthma as a heterogeneous disease which can be categorized in various ways based on such characteristics as demographics; epidemiology; natural history; histopathology; clinical manifestations; and response to therapy. However, some of the most important advances made recently in the characterization of asthma syndromes paint a picture of a common underlying etiology to at least the most prevalent form of the disease in children and perhaps in many adults.

\section{Discussion}

Viral asthma, primarily human rhinovirus-induced (HRV), is by far the most common form of active disease in children, accounting for over $80 \%$ of acute exacerbations ${ }^{2}$ and the majority of hospitalizations and clinic visits. In young infants aged under 2 years, winter viral respiratory infections play a major role in inducing the asthma phenotype. $^{3}$

It appears to be less well recognized that HRV may also account for most cases of persistent asthma. Several recent studies have shed light on this topic and document that the effects of rhinovirus-triggered asthma attacks can be sustained for weeks to months following the initial upper respiratory infection (URI). ${ }^{4}$ Evidence of HRV persistence within the airways of patients ${ }^{5,6}$ and animal models, ${ }^{7}$ suggest that persistent asthma may also be a consequence of an initial or recurrent HRV-induced airway injury. ${ }^{8}$ 
Based on these observations, and from a clinical perspective, a case can be made that at any point in time, most children and many adults with symptomatic asthma are somewhere along a continuum of partial recovery or convalescence dating back to their most recent HRV infection, with the extent of recovery being primarily dependent on the robustness of their immune response to the viral injury. It is possible to conceptualize a cycle of injury-repair over time that would explain variations in clinical severity of the disease, modified somewhat by the effects of anti-inflammatory therapy. In some cases, the acute episode does lead to a brisk immune response with quick clearance of the virus and resolution of symptoms. This would be the clinical pattern we associate with so-called "intermittent" asthma or children that wheeze "only" during acute HRV URIs. In others, the innate and adaptative immune response to the initial URI is less than adequate and fails to either clear the virus or successfully repair the damage, which then leads to persist inflammation for many weeks or months, resulting in what has been termed a "prolonged exacerbation". ${ }^{9}$ To the clinician who encounters this individual for the first time, this pattern would simply look like a "multi-trigger asthmatic" if a pre-schooler, or a "persistent" asthmatic if an adult.

A similar mechanism was proposed some years ago by Burrows ${ }^{10}$ to explain the association between frequency of acute exacerbations of chronic obstructive pulmonary disorder (COPD) and rate of decline in forced expiratory volume in 1 second $\left(\mathrm{FEV}_{1}\right)$. Since then, several studies have documented both the primary role of HRV in acute exacerbations of COPD, ${ }^{11}$ and the relationship between exacerbations and rate of decline in lung function. ${ }^{12}$ Thus, the natural histories of asthma and COPD may both be linked in a sizable percentage of patients by the number and duration of HRV-induced exacerbations.

In more severe asthma, we are positing that viral injury leads to the phenotypic characteristics of persistent asthma. HRV infections tend to reoccur fairly frequently in the pediatric population, particularly during the fall through spring months. A new URI arriving on top of a partially resolved previous infection could further overburden antiviral defenses or find an already immunologically "primed" airway, leading to a shift in clinical pattern from a mild-persistent to a more moderate or severe one. It has been our experience over many years of practice that when the fall outbreaks of HRV infections begin, it is typically not the first but the second or subsequent URIs that trigger the more serious asthma attacks. Based on recent experimental evidence on the mechanisms of viral-induced airway injury as mediated by pattern-recognition receptors, ${ }^{13}$ this "priming" effect of a viral infection could have significant implications for clinical asthma.

Another fall out from this paradigm is that it helps to explain why most children with asthma (even fairly significant persistent asthma) tend to "outgrow" the disease as they mature. It is well known that from childhood into early adolescence, older children do not experience as many URIs as their younger counterparts. Not only does the number of acute exacerbations quickly diminish with age, but the intervening period of recovery available between attacks is also extended. Thus, fewer episodes with increased recovery time results in markedly improved asthma severity. There are also host factors, particularly in terms of viral immunity, that play a significant role in modulating an individual patient's long-term pattern, but the viral etiology of the disease remains primary. Thus the role of HRV infections should not be confined to acute exacerbations but also extended to explain chronicity of the disease. This is supported by recent evidence from animal models of experimental respiratory viral infections that suggest that airway remodeling and hyperresponsiveness can persist long after the virus is cleared from the airways. ${ }^{14}$

Whatever role other environmental factors such as allergens play in the epidemiology of asthma appears trivial when faced with the accumulating evidence for the viral paradigm. ${ }^{15}$ Even previous distinctions between atopic and nonatopic asthma seem less important in light of recent endobronchial biopsy evidence that fails to distinguish between the histological presentation of atopic and nonatopic asthma. ${ }^{16}$ Knowing that the host's genotype (such as the ability to mount a robust Th2 allergic inflammatory response) plays a significant role in the viral response to injury, the primary role of HRV in driving the histopathology and clinical presentation in the large majority of asthmatics remains a valid consideration. This idea is further supported by recent advances in the biology of antiviral Th2 immune responses. The concept of virus as allergens may well lead to more widespread recognition that in most asthmatics, whether atopic or nonatopic, the disease is closely linked to an infectious viral etiology. ${ }^{17}$

\section{Implications}

What are the implications of the role that viral infections play on the natural history of asthma for clinicians who treat asthma? First and foremost is the concept of asthma as a constantly evolving disease, driven by the natural history of viral-induced airway injury and repair. To the clinician, this translates to asthma being a dynamic disease consisting of periods of acute injury followed by periods of convalescence 
that are of variable duration and intensity. This pattern can be further modulated by the effects of environmental "triggers" or by anti-inflammatory therapy. Predicting which pattern a particular patient will follow remains an elusive goal and should be the subject of further research. We believe that one of the most important questions that should be routinely asked of patients, that is not currently included in asthma control questionnaires, is "how long has it been since your last (URItriggered) exacerbation?" The answer can provide a useful index of how persistent the current exacerbation has been. The second most important question is "how many upper respiratory infections, with and without worsening asthma, have you experienced in the last ' $x$ ' number of months?' It is clear that in order to predict correctly outcomes in asthma we need clinical studies that focus on the clinical course and immunopathology of the recovery period or convalescent phase of acute asthma exacerbations.

It has recently been shown that anti-inflammatory therapy with inhaled corticosteroids has limited disease-modifying effects in asthma. One might well expect this to be the case in a primarily virus-driven condition. In a sense, viral asthma may be a disease of inadequate inflammation (less than efficient immune response to viral infection) in contrast to "too much" (small airway) inflammation. Although its clinical manifestations clearly correlate with the extent of the inflammatory processes within the airways, this response clearly fails to confine the virus to the upper airways. Thus, we are probably more likely to find success in discovering new antiasthmatic therapies if we concentrate on antiviral agents, or ways to boost the immune response to viral spread, rather than on merely suppressing airway inflammation, whether broadly with corticosteroids or narrowly with single-path antagonists of the immune response.

Another key component of the concept of asthma as an acute/subacute viral disease has substantial implications for clinical trials. Asthma clinical trials, at least those performed for the purpose of gaining regulatory approval, are typically designed around the premise that asthmatics can be neatly classified within a particular category of severity, from mild, to moderate, to severe. Several studies have documented that the patients that populate these categories do not remain static within a particular category for long, being classified as mild on some visits and more moderate or severe at another. If most asthmatics are constantly evolving over time (perhaps related to when the last viral-induced exacerbation occurred, as well as the robustness of the host response), it makes it difficult during the course of a clinical trial to ascribe changes in symptomatology or objective parameters of disease severity to a particular test treatment or intervention, even when the trial includes a placebo arm (clearly impossible without one). Since spontaneous drift in disease severity is the rule and we have no way of randomly assigning treatments based on future patterns of disease, it is not surprising that most asthma trials require large numbers of enrollees in order to minimize the effects of this variability. This intrinsic within-subject variability may also render "ethical issues" of using a placebo in asthma trials moot, since no currently available therapy has been shown to alter the course of viral-induced respiratory disease and the effect of inhaled corticosteroid therapy on rate and severity of future exacerbations is modest at best. ${ }^{18}$ Needless to say, patients with unstable disease should not be enrolled in placebo-control trials for obvious reasons.

\section{Conclusion}

In conclusion, it is generally accepted that a majority of asthma exacerbations in both children and adults are the result of viral URIs. These acute episodes are followed by a convalescent phase which can be difficult to characterize or predict in any given patient. The rate of and extent to which recovery occurs determines for the most part the asthma severity scores of the majority of patients. The frequency of viral infections should have a major role in determining disease severity and long-term patterns. Future research can productively focus on ways of characterizing airway response to viral injury, both in the acute and convalescent phases. ${ }^{19}$ Increased efforts should be directed at enhancing antiviral immune response in asthma and discovering effective and safe antiviral agents, with a vaccine against the major respiratory viral pathogens being the ultimate primary prevention strategy, although a humoral-immunity enhancing vaccine may prove futile because of the large number of HRV serotypes. A more plausible "vaccine" would enhance antiviral innate immunity, thus preventing viral attachment and/or replication.

\section{Acknowledgment}

The co-author of this paper, Michael Goldman, is now deceased.

\section{Disclosure}

The authors report no conflicts of interest in this work.

\section{References}

1. Wenzel SA. Asthma: defining of the persistent adult phenotype. Lancet 2006;368:804-813.

2. Johnston SL, Pattemore PK, Sanderswon G, et al. Community study of the role of viral infections in exacerbations of asthma in 9-11 year old children. BMJ. 1995;310:1225-1229. 
3. Wu P, Dupont WD, Griffin MR, et al. Evidence of a casual role of winter virus infection during infancy in early childhood asthma. Am J Resp Crit Care Med. 2008;178:1123-1129.

4. Kelly JT, Busse WW. Host immune response to rhinovirus: mechanisms in asthma. J Allergy Clin Immunol. 2008;122:671-682.

5. Wos M, Sanak M, Soja J, et al. The presence of rhinovirus in lower airways of patients with bronchial asthma. Am J Respir Crit Care Med. 2008; 177:1082-1089.

6. Kling S, Donninger H, Williams Z, et al. Persistence of rhinovirus RNA after asthma exacerbation in children. Clin Exp Allergy. 2005; 35:672-678.

7. Kim EY, Battaile JT, Patel AC, et al. Persistent activation of an innate immune response translates respiratory viral infection into chronic lung disease. Nat Med. 2008;14:633-640.

8. Spahn JD, Covar R. Clinical assessment of asthma progression in children and adults. J Allergy Clin Immunol. 2008;121:548-557.

9. Xepapadaki P, Papadopoulos NG, Bossios A, et al. Duration of postviral airway hyperresponsiveness in children with asthma: effect of atopy. J Allergy Clin Immunol. 2005;116:299-304.

10. Burrows B. An overview of obstructive lung diseases. Med Clin North Am. 1981;65:455-471.

11. McManus TE, Marley AM, Baxter N, et al. Respiratory viral infections in exacerbations of COPD. Respir Med. 2008;102:1575-1580.
12. Donaldson GC, Seemungal TAR, Bhowmik A, et al. Relationship between exacerbation frequency and lung function decline in chronic obstructive pulmonary disease. Thorax. 2002;57:847-852.

13. Subrata LS, Bizzintino J, Mamessier E, et al. Interactions between innate antiviral and atopic immunoinflammatory pathways precipitate and sustain asthma exacerbations in children. J Immunol. 2009;183: 2793-2800.

14. Sears MR. Epidemiology of asthma exacerbations. J Allergy Clin Immunol. 2008;122:662-668.

15. Turato G, Barbato A, Baraldo S, et al. Nonatopic children with multitrigger wheezing have airway pathology comparable to atopic asthma. Am J Respir Crit Care Med. 2008;178:476-482.

16. Karp CL. Guilt by intimate association: What makes an allergen an allergen? J Allergy Clin Immunol. 2010;25:955-960.

17. Kim EY, Battaile JT, Patel AC, et al. Persistent activation of an innate immune response translates respiratory viral infection into chronic lung disease. Nat Med. 2008;14:633-640.

18. Wilson N, Sloper K, Silverman M. Effect of continuous treatment with topical corticosteroid on episodic viral wheeze in preschool children. Arch Dis Child. 1995;2:317-320.

19. Holt PG, Strickland DH. Interactions between innate and adaptative immunity in asthma pathogenesis: New perspectives from studies on acute exacerbations. J Allergy Clin Immunol. 2010;125:963-972.
Journal of Asthma and Allergy

\section{Publish your work in this journal}

The Journal of Asthma and Allergy is an international, peer-reviewed open-access journal publishing original research, reports, editorials and commentaries on the following topics: Asthma; Pulmonary physiology; Asthma related clinical health; Clinical immunology and the immunological basis of disease; Pharmacological interventions and

\section{Dovepress}

new therapies. Issues of patient safety and quality of care will also be considered. The manuscript management system is completely online and includes a very quick and fair peer-review system, which is all easy to use. Visit http://www.dovepress.com/testimonials.php to read real quotes from published authors. 